\title{
Niedrigdosierte Doxycyclinzubereitung bei Rosazea
}

- Mit einer neuartigen Formulierung des Antibiotikums Doxycyclin können entzündliche Läsionen bei Rosazea effektiv gelindert werden. Ein Vorteil zu konventionellen Antibiotika ist die erheblich bessere Verträglichkeit.

Die Therapie mit herkömmlichen Antibiotika ist bei der Behandlung der Rosazea zwar

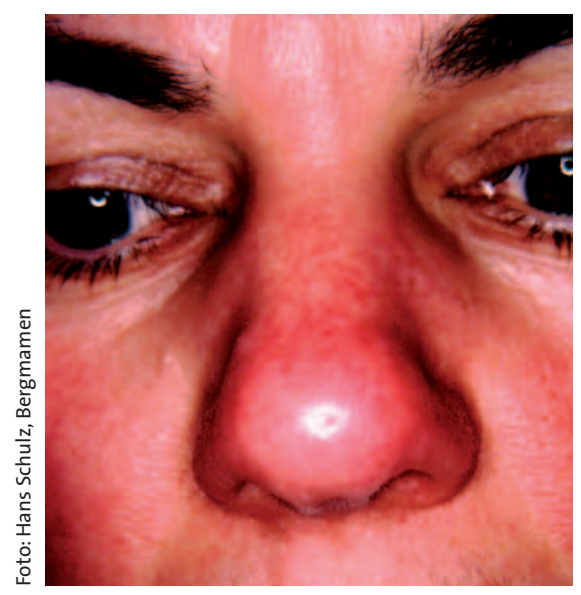

erfolgreich, allerdings sind langfristig Nebenwirkungen häufig. Auch die Entwicklung antibiotika-resistenter Bakterienstämme ist ein Problem. Abhilfe könnte hier die Entwicklung einer neuen Doxycyclinzubereitung schaffen. Dieses Antibiotikum in einer Dosierung von nur $40 \mathrm{mg}$ wird teilretardiert freigesetzt: 30 $\mathrm{mg}$ sofort, $10 \mathrm{mg}$ allmählich. Damit wird nach Ausführung von Martin Schaller, Universitätsklinik Tübingen, über 24 Stunden ein Plasmaspiegel erreicht, der für eine antimikrobielle Wirkung zu niedrig ist, aber eine antiinflammatorische Wirkung entfaltet. Genau dies ist bei Rosazea jedoch von Vorteil: Hier wird die antiinflammatorische Wirkung benötigt, und aufgrund der geringen Dosis werden keine resistenten Bakterien selektiert.

Dies zeigten auch die drei bisher mit der neuen Doxycyclinzubereitung durchgeführten

Effektive Therapie ist möglich:

Rosazea erythematosa bei einer 45-jährigen Frau.
Phase-III-Studien. In zwei Phase-III-Studien an insgesamt mehr als 500 Patienten mit mittlerer bis schwerer Rosazea konnte durch die Therapie mit dem innovativem Antibiotikum die Anzahl der entzündlichen Läsionen nach 16 Wochen im Vergleich zu Plazebo hochsignifikant verringert werden. Das Nebenwirkungsprofil lag auf Plazeboniveau. Bemerkenswert war, dass in der Verum-Gruppe kein einziger Fall einer vaginalen Kandidiose auftrat, die bei normal dosierten Antibiotika sehr häufig vorkommt.

In einer weiteren Studie, die ebenfalls 16 Wochen dauerte, wurde die innovative Doxycyclinzubereitung mit 100 mg konventionellem oralem Doxycyclin verglichen. Bezüglich der Wirksamkeit waren die beiden Gruppen vergleichbar, allerdings war das niedrig dosierte Antibiotikum hochsignifikant besser verträglich.

kam

Symposium „New Perspectives in Rosazea“ im Rahmen der 18. EADV-Tagung“. Berlin, 8.10.2009 (Veranstalter: Galderma).

\section{Licochalcon A bessert Hautirritationen}

_ Für seine Dermokosmetika hat Beiersdorf ein ungewöhnlich umfangreiches Studienprogramm aufgelegt. Nachdem in einfachen In-vitro- und komplexen Hautmodell-Untersuchungen gezeigt werden konnte, dass Licochalcon A, die Hauptkomponente eines Extraktes der chinesischen Süßholzwurzel, antiinflammatorische und antioxidative Effekte erzielt, wurde die Wirksamkeit laut Dr. Gitta Neufang, Hamburg, bei mehr als 4.000 Menschen mit unterschiedlichen akuten oder chronischen Hautirritationen überprüft: mehr als 3.100 Studienteilnehmer hatten Rosazea, mehr als 370 atopische Dermatitis und mehr als 265 Sonnenbrand.

So wendeten in einer Studie 692 Patienten mit fazialem Erythem und Rosazea begleitend über acht Wochen eine LicochalconA-Creme an: Gegenüber Studienbeginn erzielten $85,6 \%$ eine Symptomverbesserung oder -freiheit, der Anteil der Patienten, die Medikamente wie Metronidazol oder Azelainsäure benötigten, nahm von 44,7\% auf $23,4 \%$ ab und die Lebensqualität verbesserte sich von 20,4 auf 17,1 im Quality-
of-Life-Score [Schoelermann A, et al. EADV 2009, Poster].

In einer Studie zur atopischen Dermatitis mit 36 Kindern wiesen nach einer 14tägigen begleitenden Anwendung einer Licochalcon-A-Creme 93\% eine Symptomverbesserung oder -freiheit auf. Zudem nahmen der SCORAD-Wert (Score of Atopic Dermatitis) und die kutane Kolonisation mit S. aureus ab. In einer weiteren Studie, an der zwölf Menschen mit Sonnenbrand teilgenommen haben, reduzierte Licochalcon
A innerhalb von fünf Stunden im Vergleich zur Licochalcon-A-freien Vehikellotion das Erythem signifikant. Licochalcon A ist in den Eucerin ${ }^{\circledR}$-Linien „Anti-Rötung“ für Rosazea und „Omega“ für atopische Dermatitis sowie in den Sonnencremes enthalten. pe

Pressekonferenz „Licochalcon A: Ein multipotenter Wirkstoff für dermatologische und kosmetische Anwendungen - effektive therapeutische Basispflege bei atopischer Dermatitis und Rosazea, dritte Generation im Sonnenschutz". Im Rahmen der 18. EADV-Tagung, Berlin, 9.10.2009 (Veranstalter: Beiersdorf).
Neue Varianten gegen Kopfläuse

Mit seinem Zwei-Stufen-Dimeticon, das Kopfläuse, Larven und Eier ersticken lässt, hat NYDA ${ }^{\circledR}$ sich als wirksame Option zur Behandlung von Kopfläusen etabliert. Das physikalische Wirkprinzip ist gut verträglich und macht Resistenzentwicklungen unwahrscheinlich. Pohl-Boskamp hat seit dem 1.9.2009 seine Produktpalette er- weitert: Zum einen gibt es jetzt NYDA ${ }^{\circledR}$ in der günstigen Doppelpackung, falls die ganze Familie von Kopfläusen befallen ist, zum anderen ist NYDA ${ }^{\circledR}$ sensitiv ganz neu auf den Markt gekommen, das auf Duftstoffe verzichtet.

Nach Informationen von Pohl Boskamp, Hochenlockstedt 